\title{
Iris Recognition Using Fuzzy Min-Max Neural Network
}

\author{
S.S.Chowhan, Member, IACSIT, and G. N. Shinde
}

\begin{abstract}
Iris is one of the best biometric features for security applications. This paper focuses on the iris recognition and classification system and its performance in biometric identification system. The steps of iris recognition include image normalization, feature extraction and classifier. This work is an application of Patrick Simpson's fuzzy min-max neural network (FMN) Classification. Fuzzy min-max classification neural networks are built using hyperbox fuzzy sets. We performed comparative studies of different similarity measures applied to various classifiers. The feasibility of the FMN Classification Algorithm has been successfully evaluated on CASIA database with 756 images and found superior in terms of generalization and training time with equivalent testing time.
\end{abstract}

Index Terms-Biometrics, Feature extraction, Fuzzy Min-max neural network.

\section{INTRODUCTION}

In the past decade, fuzzy systems have replaced conventional technologies in many scientific applications and engineering systems, especially in control systems and pattern recognition. They can provide decision-support and expert systems with powerful reasoning capabilities bound by a minimum of rules [1]. In practice FNN's have found useful in many application fields, for instance, system modelling, system reliability analysis, pattern recognition, and knowledge engineering and so on. Based on fuzziness involved in FNN's developed since the late of 1980s [2].There are two main training schemes employed by fuzzy neural networks; In supervised learning, class labels are provided with input patterns and the decision boundary between classes that minimizes misclassification is achieved. It is often referred as pattern classification problem.

In unsupervised learning, training patterns are unlabeled and clusters of the patterns are formed with a suitable similarity measure, which is referred as clustering problem [3].The FNN models have attracted many scholars' attention. A lot of new concepts, such as innovative architecture and training rules and models about FNN's have been proposed. Hung-Hsu Tsai and Pao-Ta Yu had proposed four layer FNN for robust learning of an FNN [4]. Kwan and Cai proposed the four layer feedforward neural network When an input pattern is provided the network first fuzzifies this pattern and then computes the similarities of this pattern to all of the learned patterns. The network then reaches a conclusion by selecting the learned pattern with the highest similarity and gives a nonfuzzy output[6].

Manuscript received April 17, 2011; revised October 10, 2011.

S. S. Chowhan, Dept. of Computer Science, COCSIT, Latur, India. (e-mail: csantu_149@rediffmail.com )

G. N. Shinde, Dept. of Electronics and Computer Science, Indira Gandhi College, Nanded, India. (e-mail: shindegn@yahoo.com ).

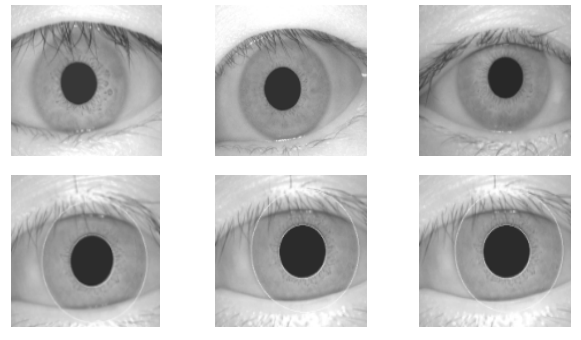

Fig. 1. Samples of Iris images with occlusion and inner and outer boundaries detected

Rui-Ping Li et al had proposed A Fuzzy Neural Network for Pattern Classification and Feature Selection which enables the classification of patterns and the selection of features and two types of learning algorithm was introduced, i.e., unsupervised learning for memory connection and supervised learning for weight connection [7]. Ability of the feature-weighted detector (FWD) network is to classify pattern and select feature.

Li Jinling had proposed Fuzzy neural network based on rectangle functions and its application Fuzzy neural network (FNN) based on rectangle functions is constructed by partitioning input space into many disjoint hyper-cubes with the same size[8]. This model is constant in each of the hyper-cubes. If and only if an input pattern drops into a hyper-cube would the corresponding pattern be memorized through coding. The FMMIS algorithm uses seed pixels to grow hyperboxes, and a criterion of homogeneity for controlling the size of these hyperboxes. If the homogeneity criterion is satisfied, a hyperbox could be expanded from a given size to any other size by including a new seed pixel which was used faster segmentation [10]. Juan Wachs et al had focused on automated method of segmentation of faces in color images using fuzzy min-max neural network which automatically segments the skin colors of the face in a Hue Saturation Value color model [11]. Cheng, H.D. Cui, M. had proposed Mass lesion detection with a fuzzy neural network approach to detect malignant mass lesions on mammograms [12]. Suliman M Mohamed et al had used fuzzy neural network for Automatic Fingerprint Classification this classifier was trained and tested on 4000 images in NIST-4 database [13].

\section{FEATURES OF FuZZY SET AND MEMBERShiP FunCTION}

Fuzzy sets were introduced by Zadeh as a means of representing and manipulating data that were not precise, but rather fuzzy [14]. Zadeh's extension of set theory provided a mechanism for representing linguistic constructs such as "little more"," very tall", "very far" and it gave pattern classification and control engineers the ability to measure the degree to which a pattern was present or a situation was occurring. The values assigned to the elements of the 
universal set fall within a specified range and indicate the membership grade of these elements in the set. Larger values denote higher degrees of set membership [9]. Such a function is called membership function, and the set defined by it a fuzzy set. The most common notation are employed to denote the membership functions of a fuzzy set $\mathrm{A}$ is.

$$
A=\left\{x, \mu_{A}(x) \mid x \in X\right\}
$$

Each fuzzy set is completely and uniquely defined by one particular membership function. The membership value $\mu_{A}(x)$ describes the degree to which the element belongs to the set $\mathrm{A}$, where $\mu_{A}(x)=0$ represents lowest membership and $\mu_{A}(x)=1$ represents highest membership.

In pattern classification using fuzzy sets, class fuzzy sets are created with the nature of input patterns applied to the fuzzy-neural networks. This is the training process. In recall phase the membership value of input pattern is calculated in all class fuzzy sets and the class label of a fuzzy set is given to the input pattern in which it acquires highest membership.

\section{A. Normal Fuzzy set}

A fuzzy set defined by its membership function must be a normal fuzzy set. A normal fuzzy set is defined as a set in which at least one element from the universe has membership value to be highest is called as normal when $h(A)=1$. Fig. 2 shows a normal fuzzy set, it is called subnormal when $h(A)<1$, that is.

$$
h(A)=\sup _{x \in X} A(x)
$$

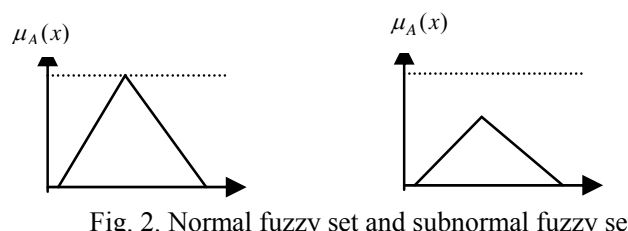

\section{B. Fuzzy set operations}

The three basic operations on classical sets are union, intersection and complement can be generalized in more than one way [10] which are usually referred to as standard fuzzy set operations, has special significance in fuzzy set theory. These operations are defined as follows.Union of two fuzzy sets $\mathrm{A}$ and $\mathrm{B}$ is a fuzzy set $\mathrm{C}$ is defined as

$$
\mu_{C}(x)=\mu_{A}(x) \vee \mu_{B}(x)=\max \left[\mu_{A}(x), \mu_{B}(x)\right] ; x \in X
$$

Intersection of two fuzzy sets A and B is a fuzzy set $\mathrm{C}$ is defined as

$$
\mu_{C}(x)=\mu_{A}(x) \wedge \mu_{B}(x)=\min \left[\mu_{A}(x), \mu_{B}(x)\right] ; x \in X
$$

Absolute and relative complements are defined as

$$
\mu_{A}(x)=1-\mu_{A}(x) \text { for all } x \in X
$$

A relative complement of $\mathrm{A}$ with respect to $\mathrm{B}$ denoted by $\mathrm{B}-\mathrm{A}$ is defined by $\mu_{A}(x)=\mu_{B}(x)-\mu_{A}(x)$.

\section{TOPOLOGY OF FUZZY MIN-MAX NEURAL NETWORK}

The fuzzy min-max neural network consists of three-layer as shown in the figure 2. The input layer $F_{A}=\left(a_{1}, a_{2} \ldots \ldots a_{n}\right)$ has $n$ processing elements, one for each of the $n$ dimensions of the input pattern $A_{h}$. There are two sets of connections between each input node and each of the $m$ hyper box fuzzy set nodes found in the layer $F_{B}=\left(b_{1}, b_{2} \ldots \ldots . b_{n}\right)$. These dual connections are adjusted using the fuzzy min-max classification learning algorithm. There are two sets of connections that emanate from $F_{A}$ and neighbor of the $j^{\text {th }} F_{B}$ node-the min vector $V_{j}$, and the max vector $W_{j}$. The connections between the $F_{B}$ nodes and the $p$ output nodes $F_{C}=\left(c_{1}, c_{2} \ldots \ldots . c_{p}\right)$ are binary valued and are determined as each $F_{B}$ node is added during learning. Each $F_{C}$ node represents a pattern class [5].

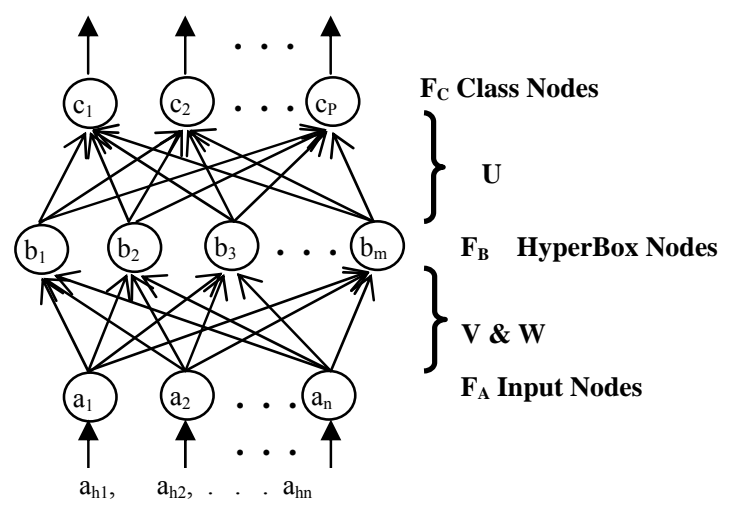

Fig. 3. Topology of fuzzy min-max neural network

Let the $j^{\text {th }}$ hyper box fuzzy set, $B_{j}$, be defined by the order set:

$$
B_{j}=\left\{X, V_{j}, W_{j}, f\left(X, V_{j}, W_{j}\right)\right\}
$$

Using equation 6 the aggregate fuzzy set defines the $K^{\text {th }}$ pattern class $C_{k}$ is defined as

$$
C_{k}=\bigcup_{j \in k} B_{j}
$$

where $K$ is the index set of those hyperboxes associated with class $\mathrm{k}$. Note that the union operation in fuzzy sets is typically the max of all of the associated fuzzy set membership functions.

\section{A. Memebership function}

The membership function for the $j^{\text {th }}$ hyperbox $b_{j}\left(A_{h}\right)$, $0 \leq b_{j}\left(A_{h}\right) \leq 1$, must measure the degree to which the $h$ th input pattern $A_{h}$ falls outside of the hyperbox $B_{j}$ formed by the min point $V_{j}$ and the max point $W_{j}$. As $A_{h}$ approaches 1 , and when the point is contained within the hyperbox, $b_{j}\left(A_{h}, V_{j}, W_{j}\right)=1$. The resulting membership function is defined as

$$
\begin{aligned}
b_{j}\left(A_{h}\right)= & \frac{1}{2 n} \sum_{i=1}^{n}\left[\max \left(0,1-\max \left(0, \gamma \min \left(1, a_{h i}-w_{j i}\right)\right)\right)\right. \\
& \left.+\max \left(0,1-\max \left(0, \gamma \min \left(1, v_{j i}-a_{h i}\right)\right)\right)\right]
\end{aligned}
$$

where $A_{h}=\left(a_{h 1}, a_{h 2}, \ldots . . a_{h n}\right) \in I^{n}$ is the $h$ th input pattern, 
$V_{j}=\left(v_{j 1}, v_{j 2}, \ldots \ldots . v_{j n}\right)$ is the min point and max point for $b_{j}$ is $w_{j}=\left(w_{j 1}, w_{j 2}, \ldots \ldots . w_{j n}\right)$ and $\gamma$ is the sensitive parameter $0 \leq \gamma \leq 1$, which governs how fast the membership value decreases outside the hyperbox as $A_{h}$ and $b_{j}$ increases.

\section{FuZZY Min-MAX LEARNING ALGORITHM}

The supervised FMM learning algorithm consists of three steps:

Expansion: Identify the hyperbox that can expand and expand it. If an expandable hyperbox cannot be found, add a new hyperbox for that class.

Overlap Test: Determine if any overlap exists between hyperboxes from different classes.

Contraction: If overlap between hyperboxes that represent different classes does exist, remove the overlap by minimally adjusting each of the hyperboxes

\section{A. Hyperbox Expansion}

Given the $h$ th training pair $\left(A_{h}, d_{h}\right)$, find the hyperbox $B_{j}$ which provides the highest degree of membership, allows expansion if necessary and represents the same class as $d_{h}$ the degree of membership is measured using equation 8 . For the hyperbox $B_{j}$ to expand to include $A_{h}$ the following constraint must be met.

$$
n \theta \geq \sum_{i=1}^{n}\left(\max \left(w_{j i}, a_{h i}\right)-\min \left(v_{j i}, a_{h i}\right)\right)
$$

If the expansion criterion has been met for hyperbox $B_{j}$ min point of the hyperbox is adjusted using the equation

$$
v_{j i}^{\text {new }}=\min \left(v_{j i}^{\text {old }}, a_{h i}\right)
$$

and the max point is adjusted using the equation

$$
w_{j i}^{\text {new }}=\max \left(w_{j i}^{\text {old }}, a_{h i}\right)
$$

\section{B. Hyperbox Overlap Test}

Considering that the hyperbox $B_{j}$ was expanded from the above step and that the hyperbox $B_{k}$ represents another class and it is to be tested for possible overlap class. Assuming $\delta^{\text {old }}=1$ initially, the four test cases is satisfied for each of the $n$ dimension.

$$
\begin{aligned}
& \text { case } 1: v_{j i}<v_{k i}<w_{j i}<w_{k i}, \\
& \text { case } 2: v_{k i}<v_{j i}<w_{k i}<w_{j i}, \\
& \text { case 3: } v_{j i}<v_{k i} \leq w_{k i}<w_{j i}, \\
& \text { case } 4: v_{k i}<v_{j i} \leq w_{j i}<w_{k i} .
\end{aligned}
$$

\section{Hyperbox Contraction}

If $\Delta>0$, then the $\Delta$ th dimensions of the two hyperboxes are adjusted. To determine the proper adjustment to make, the same four cases are studied.

Case $1: v_{j i \Delta}<v_{k \Delta}<w_{j \Delta}<w_{k \Delta}, \quad w_{j \Delta}^{\text {new }}=v_{k \Delta}^{\text {new }}=\frac{w_{j \Delta}^{\text {old }}+v_{k \Delta}^{\text {old }}}{2}$

Case 2: $v_{k \Delta}<v_{j \Delta}<w_{k \Delta}<w_{j \Delta}, w_{k \Delta}^{\text {new }}=v_{j \Delta}^{\text {new }}=\frac{w_{k \Delta}^{\text {old }}+v_{j \Delta}^{\text {old }}}{2}$

Case 3i $v_{j \Delta}<v_{k \Delta}<w_{k \Delta}<w_{j \Delta}$, and $\left(w_{k \Delta}-v_{j \Delta}\right)<\left(w_{j \Delta}-v_{k \Delta}\right)$, $v_{j \Delta}^{\text {new }}=w_{k \Delta}^{\text {new }}$

Case 3ii : $v_{j \Delta}<v_{k \Delta}<w_{k \Delta}<w_{j \Delta}$, and $\left(w_{k \Delta}-v_{j \Delta}\right)>\left(w_{j \Delta}-v_{k \Delta}\right)$, $w_{j \Delta}^{\text {new }}=v_{k \Delta}^{\text {old }}$.

Case $4 \mathrm{i}: v_{k \Delta}<v_{j \Delta}<w_{j \Delta}<w_{k \Delta}$ and $\left(w_{k \Delta}-v_{j \Delta}\right)<\left(w_{j \Delta}-v_{k \Delta}\right)$, $w_{k \Delta}^{\text {new }}=v_{j \Delta}^{\text {old }}$.

Case 4ii : $v_{k \Delta}<v_{j \Delta}<w_{j \Delta}<w_{k \Delta}$ and $\left(w_{k \Delta}-v_{j \Delta}\right)>\left(w_{j \Delta}-v_{k \Delta}\right)$, $v_{k \Delta}^{\text {new }}=w_{j \Delta}^{\text {old }}$.

\section{IRIS SEGMENTATION AND FEATURE EXTRACTION}

Iris Segmentation plays very important role for detecting the iris patterns, Segmentation is to locate valid part of the iris for iris biometric [15], [18]. Finding the inner and outer boundaries (pupillary and limbic) are as shown in the Fig 1. Localizing it's upper and lower eyelids if they occlude, and detecting and excluding any overlaid occlusion of eyelashes and their reflection. The best known algorithm for iris segmentation is Daugman's intergro-differential operator to find boundaries of iris as defined.

$$
\max _{(r, x o, y o)}\left|G_{\sigma}(r)^{*} \frac{\partial}{\partial r} \oint_{r, x o, y o} \frac{I(x, y)}{2 \pi r} d s\right|
$$

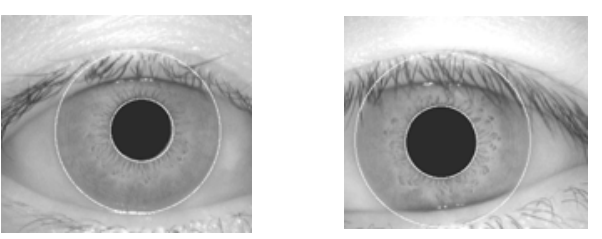

Fig. 4 Inner and Outer boundaries are detected.

Iris has a particularly interesting structure and provides rich texture information. Here we have implemented principal component analysis method for feature extraction which captures local underlying information from an isolated image. The result of this method yields high dimension feature vector. To improve training and recalling efficiency of the network, here we have reduced dimensionality of the feature vector by using Singular value decomposition (SVD) method. SVD is a method for identifying and ordering the dimensions along which the feature exhibit the most variation [19].This can be described as

$$
X=U T V^{T}
$$

the covariance matrix is be defined as:

$$
C=\frac{1}{n} X X^{T}=\frac{1}{n} U T^{2} U^{T}
$$


$U$ is a $n \times m$ matrix. SVD performs repetitively order the singular values in descending order, if $n<m$, the first $n$ columns in $U$ corresponds to the sorted eigenvalues of $C$ and if the first $m$ corresponds to the sorted non-zero eigenvalues of $C$. The transformed data can thus be written as:

$$
Y=\widetilde{U}^{T} X=\widetilde{U}^{T} U T V^{T}
$$

where $\widetilde{U}^{T} U$ is a simple $\mathrm{n} \times \mathrm{m}$ matrix which is one on the diagonal and zero. Hence Equation 13 is decomposition of equation 13 .

\section{EXPERIMENTAL RESULT}

CASIA Iris Image Database includes 756 iris images from 108 eyes. For each eye, 7 images are captured in two sessions, where three samples are collected in the first session and four samples in second. For each iris class, we choose two samples from each session for training and remaining as testing samples. The timing analysis of training and recall, recognition rates in terms of number of hyperboxes, are as shown in Table.I and Table.II

\begin{tabular}{llll}
\multicolumn{4}{c}{ TABLE I: TIMING ANALYSIS } \\
\hline \hline $\begin{array}{l}\text { Training Time in } \\
\text { Seconds }\end{array}$ & $\begin{array}{l}\text { Recall time } \\
\text { In seconds }\end{array}$ & $\begin{array}{l}\text { Neurons in output } \\
\text { layer/Hyperboxes }\end{array}$ \\
\hline FNN & 7.2367 & 145.5573 & 535 \\
\hline FMN & 6.1303 & 125.1188 & 520 \\
\hline \hline
\end{tabular}

TABLE II: RECOGNITION RATES

\begin{tabular}{lll}
\hline \hline Methodology & Recognition rate & Classifier \\
\hline Daugman & 99.25 & HD,SVM \\
\hline Wildes & 97.43 & Normalized Correlation \\
\hline Y. Wang & 99.57 & WED \\
\hline Ali & 95.20 & HD,WED \\
\hline FMN & 95.68 & FMN \\
\hline \hline
\end{tabular}

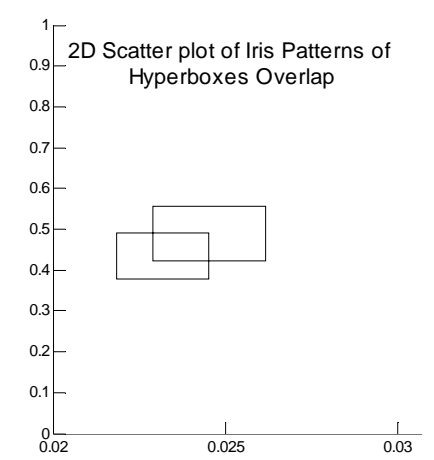

Fig. 5(a) Scatter plot Iris Patterns of Hyperboxes Overlap

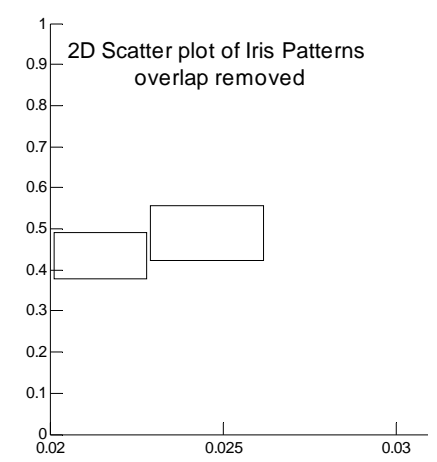

Fig. 5(b) 2D Scatter plot Iris Patterns Overlap removed

\section{CONCLUSION}

A Supervised Neural Network Classifier that utilizes hyper box fuzzy set for Iris Recognition. The performance of FMN is analyzed and tested on CASIA database and also compared with other methodology. Hence the hyperboxes are created less in FMN. Therefore training and recall time is less than FNN. And also the recognition rates are compared in Table II.

\section{REFERENCES}

[1] Chung-Ju Chang, et al., "Fuzzy Neural congestion control for integrated voice and data DS-CDMA/FRMA cellular networks," IEEE Journal On Selected Areas In Communications”, vol.18, no.2, pp. 283-293, February 2000.

[2] Lee M. R., Rhee H., Cho K. \& Cho B. J., "Self-tuning of the fuzzy inference rule by integrated method, "Journal of Intelligent \& robotic systems, vol. 33, no. 3, 313-327, 2002.

[3] Pradeep M. Patil, S N Kulkarni, D D Doye and U V Kulkarni "Modular General Fuzzy Hypersphere Neural Network," in Proc. 4th WSEAS/IASME Int. Conf. on System Science and Simulation in Engineering, Tenerife, Spain, December 16-18, 2005, pp211-216.

[4] Hung-Hsu Tsai and Pao-Ta Yu "On the Optimal Design of Fuzzy Neural Networks with Robust Learning for Function Approximation," IEEE TRANS. Systems, Man, And Cybernetics, Vol.30, no.1, February 2000.

[5] Patrick K. Simpson, "Fuzzy Min-Max Neural Networks-Partl: Classification," IEEE Trans on Neural Networks, vol. 3, no.5, pp.776-787.

[6] Hon Keung Kwan and Yaling Cai “A Fuzzy Neural Network and its Application to Pattern Recognition," IEEE Transactions on Fuzzy Systems, Vol. 2. No. 3, August 1994.

[7] Rui-Ping Li.et.al , "A Fuzzy Neural Network for Pattern Classification and Feature Selection," Dept. of Computer Science, Meiji University, Higashimita, Tama-ku, kawasaki, 214, Japan.

[8] Li Jinling, "Fuzzy neural network based on rectangle functions and its application," ICISE, Hangzhou, China, December 4 -6, 2010.

[9] George J. Klir and Bo Yuan, "Fuzzy Sets and Fuzzy Logic Theory and Applications," PHI, 2002, pp.

[10] Pablo A. Estévez et.al "Fuzzy Neural Network for Image Segmentation," Dept. of Electrical Engineering University of Chile.

[11] J. Wachs, H. Stern and M. Last, "Color face segmentation using a fuzzy min-max neural network,” Int. J. Image an Graphics, Vol. 2, No. 4, pp. 587-601, 2002.

[12] Cheng, H.D. Cui, M., "Mass lesion detection with a fuzzy neural network," Dept. of Comput. Sci., Utah State Univ., Logan, UT, USA, ICASSP, April 6- 10, 2003.

[13] Suliman M Mohamed and Henry O Nyongesa, "Automatic Fingerprint Classification System Using Fuzzy Neural Techniques," Sheffield Hallam University Sheffield 2002.

[14] L. A. Zadeh, "Fuzzy Sets", Department of Electrical Engineering and Electronics Research Laboratory, University of California, Berkeley, California.

[15] J. Daugman, "High Confidence Visual Recognition of Persons by a Test of Statistical Independence," IEEE Trans. Pattern Anal. Machine Intell., vol. 15, no. 11, pp. 1148-1161, Nov. 1993.

[16] R. P. Wildes, "Iris Rcognition: An Emerging Biometric Technology," In Proceeding of the IEEE, Piscataway, NJ, USA, 1997, 85:1348-1363.

[17] The centre of Biometrics and security Research, CASIA Iris image Database, http://www.sinobiometrics.com.

[18] J. G. Daugman, "How Iris Recognition Works," Proc. International Conference on Image Processing, Vol. 1, 2002, pp.33-36.

[19] Madsen, R., Hansen, L., \&Winther, O. "Singular value decomposition and principal component analysis," ISP Technical report, 2004.

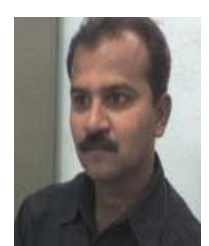

Santosh S. Chowhan received the M.Sc.(CS) degree from Dr. BAM University, Aurangabad in the year 2000. He received the M.Phil. Degree in Computer Science from Y.C.M.O. University, Nashik in the year 2008.

$\mathrm{He}$ is currently working as lecturer in the College of Computer Science and Information Technology, Latur, Maharastra. His current research interests include various aspects of Neural Networks and Fuzzy Logic, Pattern Recogntion and Biometrics. 


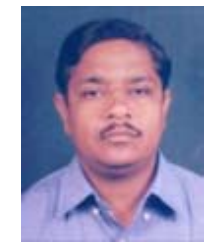

Ganeshchandra N. Shinde received M. Sc. \& Ph.D. degree from Dr. B.A. M. University, Aurangabad..

$\mathrm{He}$ is currently working as Principal in Indira Gandhi College, Nanded, Maharashtra, INDIA. He has awarded Benjongi Jalnawala award for securing highest marks at B.Sc. He has published 27 papers in the International Journals and presented 15 papers in International Conferences. In his account one book is published, which is reference book for different courses.

He is member of different academic \& professional bodies such as ANAS (Jordan). He is in reviewer panel for different Journals such as IEEE (Transactions on Neural Networks), International Journal of Physical Sciences (U.S.A.), Journal of Electromagnetic Waves and Applications (JEMWA, U.S.A.). He was the Chairperson for F-9 session of International Conference on Computational and Experimental Science \& Engineering which was held at Honolulu, U.S.A. He is member of Management Council \& Senate of S.R.T.M. University, Nanded, INDIA. His research interest includes Filters, Image processing, Pattern recognition, Fuzzy Systems, Neural Network and Multimedia analysis and retrieval system. 